\title{
Local Electric Field in Mass-Impregnated HVDC Cables
}

\author{
Gunnar Håkonseth $^{1,2}$, Erling Ildstad ${ }^{1}$, and Knut Magne Furuheim ${ }^{2}$ \\ ${ }^{1}$ Department of Electric Power Engineering, \\ Norwegian University of Science and Technology (NTNU), NO-7491 Trondheim, Norway \\ ${ }^{2}$ Nexans Norway AS, PO Box 42, NO-1751 Halden, Norway
}

\begin{abstract}
Detailed knowledge of the electric field in the insulation of mass-impregnated HVDC cables is important in order to understand their breakdown mechanisms and to improve their design. When calculating the local electric field, the wrapped structure of paper and impregnating oil needs to be taken into account. This gives information that is lost when the insulation is treated as a single, homogeneous material, which is done when the macroscopic electric field is calculated.
\end{abstract}

We have modeled mass-impregnated cable insulation with finite element analysis software and subsequently calculated the local electric field with the finite element method. The wrapped structure of paper and impregnating oil was taken into account.

In our model, the magnitude of the local field in the paper was up to twice as high as the magnitude of the macroscopic field. Field enhancements came from oilfilled butt gaps, and particularly from their edges. The geometry of the insulation influenced the magnitude of the field enhancements.

\section{Introduction}

High voltage direct current (HVDC) cables for long lengths are traditionally insulated with mass-impregnated paper [1], and this insulation technique is still being used for new, long transmission links [2]. The insulation consists of paper strips that are helically wrapped around the conductor of the cable. The width of the strips and pitch angle are tuned to leave a gap between adjacent paper windings in order to accommodate bending of the cable. These gaps are called "butt gaps". The windings of each paper layer are staggered with respect to the previous layer so that the butt gaps are not positioned directly above each other. The butt gaps, as well as the porous structure of the paper itself, are filled with "mass", a high-viscosity impregnation compound consisting of mineral oil with additives and thickeners.

Knowledge of the electric field in the insulation is key to understanding breakdown mechanisms of such cables, which again is important when designing cables for higher voltages than the current state of the art. It has been common to view the combination of paper and oil as a single, homogeneous material, disregarding the layered structure and the butt gaps. Experimental, analytical, and numerical techniques have then been used to find the macroscopic electric field. The macroscopic electric field and its gradient are always pointing from the conductor to the insulation screen, or in the opposite direction. The macroscopic approach is useful in order to investigate effects of different service conditions and situations that occur during the operation of HVDC mass-impregnated cables. Such conditions and situations can be temperature gradients and rapid voltage changes $[3,4,5]$.

However, the macroscopic approach neglects the effects of the wrapped geometry and the different electric properties of paper and oil. It masks the variations in the electric field due to the heterogeneity of the insulation, and therefore local peaks in the electric field strength are not discovered. The macroscopic approach also disregards the electric field components other than the radial component. A more detailed approach is necessary in order to reveal critical spots in the insulation where the local electric field is very high. Calculations of the local electric field need to take into account the geometry of the insulation on a millimeter scale and below. This includes paper thickness, butt gap size, and the interfaces between the paper layers.

This paper presents calculations of the local electric field at stationary, direct current (DC) conditions done with the finite element method (FEM). It shows spots where the electric field is higher than in its surroundings, indicating critical spots in the insulation.

\section{Theory}

If the electric potential $V$ is known, the electric field $E$ at DC conditions is

$$
\vec{E}=-\nabla V
$$

The basis for calculation of the potential is, together with the equation (1) above, the continuity equation

$$
\nabla \vec{J}=0
$$

and Ohm's law

$$
\vec{J}=\sigma E .
$$

$\vec{J}$ is current density and $\sigma$ is conductivity.

A Maxwell capacitor consists of two parallel plates with two, parallel dielectric slabs $a$ and $b$ with conductivities 
$\sigma_{a}$ and $\sigma_{b}$, respectively. At steady state, the field distribution will be resistive and independent of permittivity. The electric field $E$ is

$$
E_{a}=U \frac{\sigma_{b}}{d_{a} \sigma_{b}+d_{b} \sigma_{a}}
$$

where $U$ is the voltage between the plates and $d_{a, b}$ are the thicknesses of the dielectrics [6]. If now $a$ is impregnated paper, $b$ is oil, and $\sigma_{\text {oil }}=k \sigma_{\text {paper }}$ where $k$ is some constant, equation (4) becomes

$$
E_{\text {paper }}=\frac{U}{d_{\text {paper }}+d_{\text {oil }} / k} .
$$

The relationship between the electric fields in the dielectrics becomes

$$
E_{\text {paper }}=k E_{\text {oil }} .
$$

This predicts that if $k \gg 1$, meaning $\sigma_{\text {oil }} \gg \sigma_{\text {paper }}$, almost all the potential drop will be through the paper.

\section{Method}

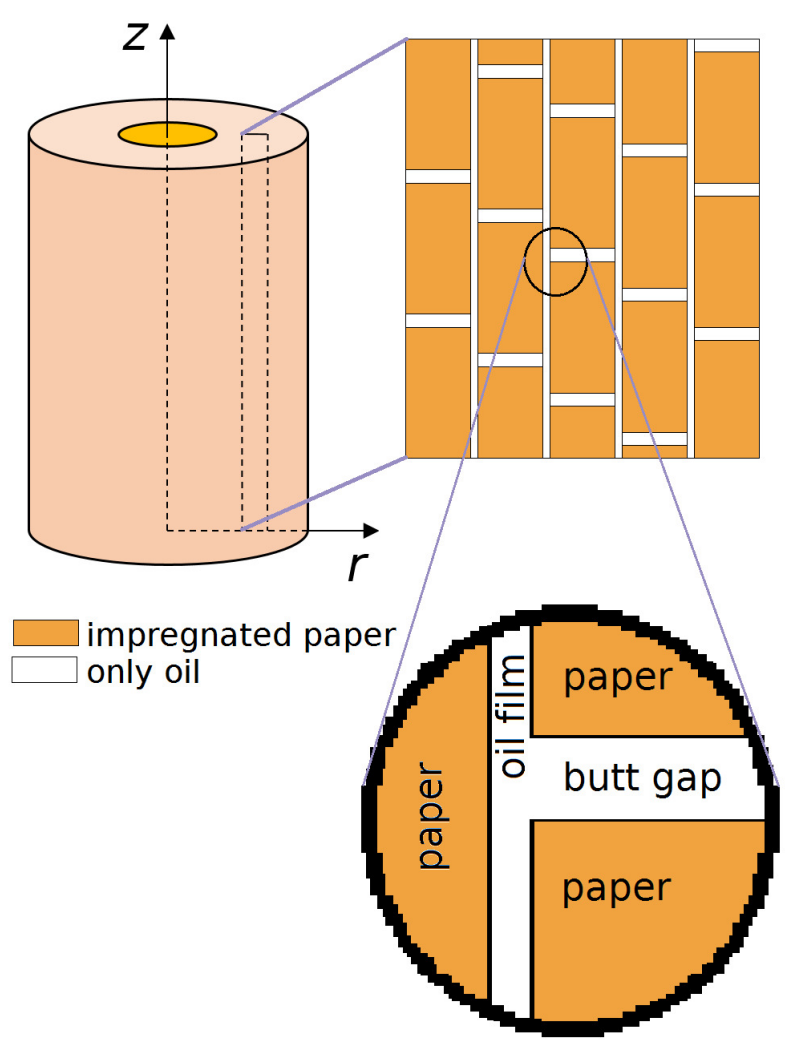

Fig. 1 - Model. Note that the detailed part of the model is heavily stretched in the $r$ direction in order to enhance visibility of the narrow parts. Some of the subsequent figures are stretched similarly.

A two-dimensional cylindrically symmetric model of part of a paper-oil insulation system was created with the finite element analysis (FEA) software Comsol Multiphysics version 5.2 on a laptop computer. The symmetry axis and the radial axis are hereafter called the $z$ and $r$ axis respectively. A sketch of the model and its cylindrical coordinate system is shown in Figure 1. The cylindrical symmetry neglected the helical structure of the insulation by assuming that the paper strips and butt gaps are closed rings instead of helices.

The model consisted of a small section of the insulation of a $450 \mathrm{kV}$ mass impregnated HVDC cable with conductor cross-section $1600 \mathrm{~mm}^{2}$, conductor radius (including semi-conductive screen) $23.2 \mathrm{~mm}$, and insulation thickness $19.2 \mathrm{~mm}$. The macroscopic field of such a cable is previously studied [5].

The paper strips in our model were $20 \mathrm{~mm}$ wide and $100 \mu \mathrm{m}$ thick. Neighboring layers of paper strips were staggered $30 \%$. The butt gaps were $2 \mathrm{~mm}$ wide. The interfaces between the paper layers were modelled as oil films. These oil films can be seen in Fig. 1 as long, vertical, white rectangles. Calculations were done with oil film thickness $d_{\text {film }}$ of $1 \mu \mathrm{m}$ and $10 \mu \mathrm{m}$. Calculations were also done without any oil films between the papers.

Limitations in computing memory and speed prohibited analysis of more than a small section of the insulation. The section contained five layers of paper in order to reveal effects of the staggering. This corresponded to a section thickness $d_{\text {section }}$ of $0.500-0.540 \mathrm{~mm}$. The length of the section along the $z$ axis corresponded to the width of three paper strips and two butt gaps, which was $64 \mathrm{~mm}$. The section was placed against the conductor. Thus, since the conductor radius was $23.2 \mathrm{~mm}$, the left and right edges of the model were at $r=23.2 \mathrm{~mm}$ and $r=23.2+d_{\text {section }}$, respectively.

The paper was modeled as a solid, homogeneous material. The internal microstructure and natural residues from the wood were looked apart from. This implies that the paper in our model was to be understood as impregnated paper, i.e. paper whose pores and interstices between fibers were filled with oil. Consequently, any variations in the electric field due to the porous structure of the paper were disregarded. Furthermore, all surfaces and edges were modeled as perfectly smooth, neglecting the surface roughness of the paper. It follows that the transition between the $100 \mu \mathrm{m}$ paper strip edges and the $20 \mathrm{~mm}$ paper surfaces were modeled as right-angled corners. It was assumed that the oil completely wetted the surfaces of the impregnated paper, causing close contact between the oil films and the papers. The model was free of cavities that usually form in mass-impregnated cable insulation during cooling [7, 8]. It was also free from other imperfections and contaminations.

The conductivity of impregnated paper was set to follow the relation

$$
\sigma=A \exp \left(-\frac{a}{T}+b|\vec{E}|\right)
$$

with $a=1.10 \times 10^{4} \mathrm{~K}, b=0.034 \mathrm{~mm} / \mathrm{kV}$, and $A=$ $4.3 \mathrm{~S} / \mathrm{m}$. The conductivity of oil was set to be 100 times larger than the conductivity of impregnated paper, meaning $A=430 \mathrm{~S} / \mathrm{m}$ for oil [3]. The temperature was 
set to $50{ }^{\circ} \mathrm{C}$ across the whole insulation, leaving the effect of temperature gradients out of the analysis.

The finite element mesh consist of rectangular elements. The longitudinal element size was $20 \mu \mathrm{m}$ for all the elements. The radial element size was $0.2 \mu \mathrm{m}$ for the elements in the oil films and in the papers next to the oilpaper interfaces. It was exponentially increasing towards the middle of the paper strips, where the radial elements size was $6 \mu \mathrm{m}$.

The steady-state, macroscopic field in a HVDC cable varies across the insulation. In a small domain close to the conductor, it can be approximated to

$$
E(r)=\alpha\left(r-r_{\mathrm{i}}\right)+\beta .
$$

$r_{\mathrm{i}}$ is the conductor radius including the semi-conductive screen, and $\alpha$ and $\beta$ are constants. With constant temperature across the insulation in a cable like the one we have modeled, $\alpha=0.6 \mathrm{kV} / \mathrm{mm}^{2}$ and $\beta=27.8 \mathrm{kV} / \mathrm{mm}$ in the innermost part of the insulation [5]. By integrating equation 8 , we get the potential

$$
U(r)=-\frac{1}{2} \alpha\left(r-r_{\mathrm{i}}\right)+\beta .
$$

With $\alpha$ and $\beta$ as mentioned above, this potential was used as boundary condition at the left and right boundaries of the model. This corresponded to a macroscopic field of $28 \mathrm{kV} / \mathrm{mm}$. The boundary condition used for the top and bottom boundaries of the model were that no current could cross the boundary, meaning

$$
\hat{n} \cdot \vec{J}=0
$$

where $\hat{n}$ is the normal vector to the boundary.

The FEA software on the laptop computer used FEM to calculate the potential $U$ and the field $\vec{E}$ by solving equations (1), (2), and (3) together with the boundary conditions (9) and (10).

\section{Results}

\subsection{Field enhancement from butt gaps}

Figure 2 shows the electric field in the whole model. The paper above and below the butt gaps had an electric field strength of $28 \mathrm{kV} / \mathrm{mm}$. The paper that were directly to the left or right of a butt gap had 24-25\% higher electric field. The field in the oil was $0.8-0.9 \mathrm{kV} / \mathrm{mm}$ in the films $1.1-1.2 \mathrm{kV} / \mathrm{mm}$ in the butt gaps. This gave a value of approximately 30 for $k$ in equations (5) and (6).

This field enhancements reached several paper layers away from the butt gaps. When our model was extended to include twelve layers of paper, areas of field enhancement coincided with each other. This is shown in Figure 3.

\subsection{Field enhancement from paper edges}

Paper edges, which also are butt gap edges, are defined in Figure 4. These edges induced field enhancements

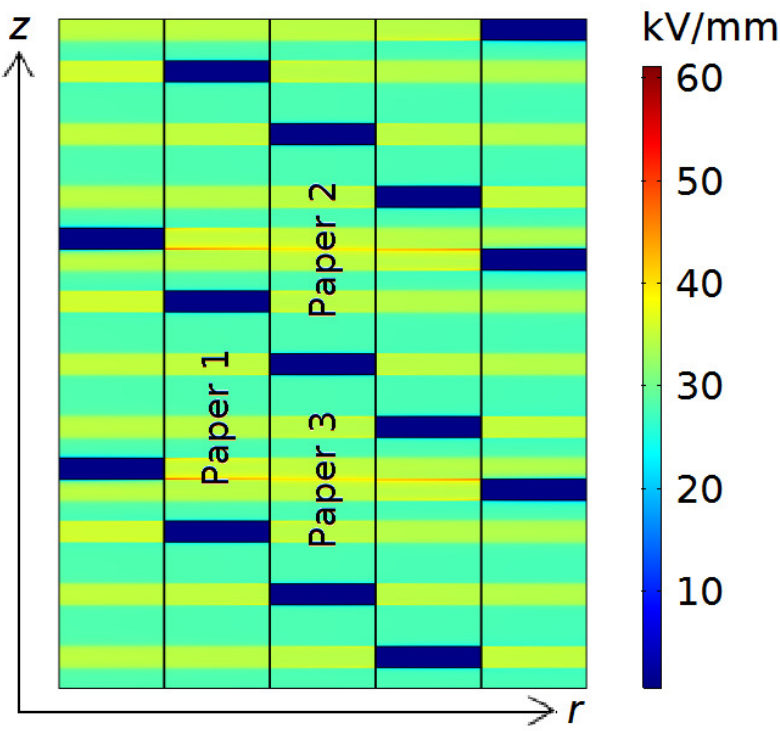

Fig. 2 - Electric field strength with $d_{\text {film }}=1 \mu \mathrm{m}$. The dark blue areas are oil films (long, vertical) and butt gaps, and the rest is paper. Note that the figure is stretched in the $r$ direction: The scales of the axes differ by a factor 100 so that the thickness of the papers, butt gaps, and oil films are exaggerated.

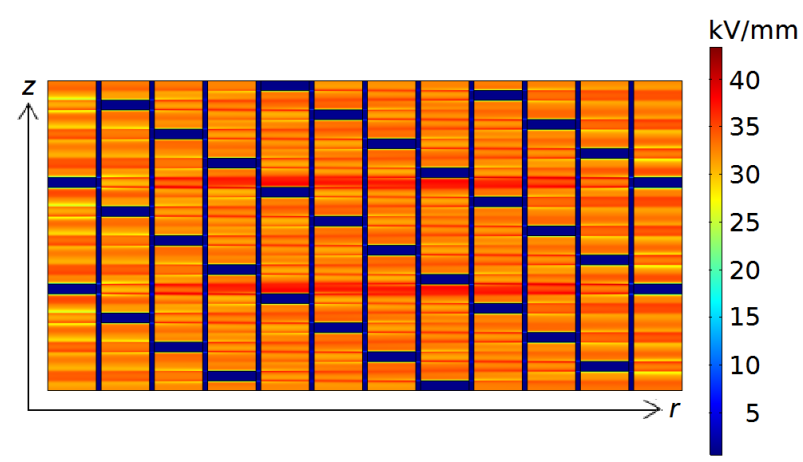

Fig. 3 - Electric field strength with $d_{\text {film }}=10 \mu \mathrm{m}$. Note that the figure is stretched in the $r$ direction: The scales of the axes differ by a factor 50 so that the thickness of the papers, butt gaps, and oil films are exaggerated.

that were different from the field enhancements described above. The cause of the edge-induced field can be understood by observing Figure 5, where the butt gap "pushed away" equipotential lines while Paper 3 did not. The equipotential lines were concentrated at the corner of the butt gap, and this concentration constituted an edgeinduced field enhancement.

The edge-induced field enhancements can be seen as peaks in Figure 6 and Figure 7.

The edge-induced enhancements were stronger in the longitudinal than in the radial direction, but the major component of the total electric field was still radial in the peak of the enhancements. The effect of the edges was mainly seen close to the paper surface, which also was close to the edge.

The edge-induced field enhancements coincided with 


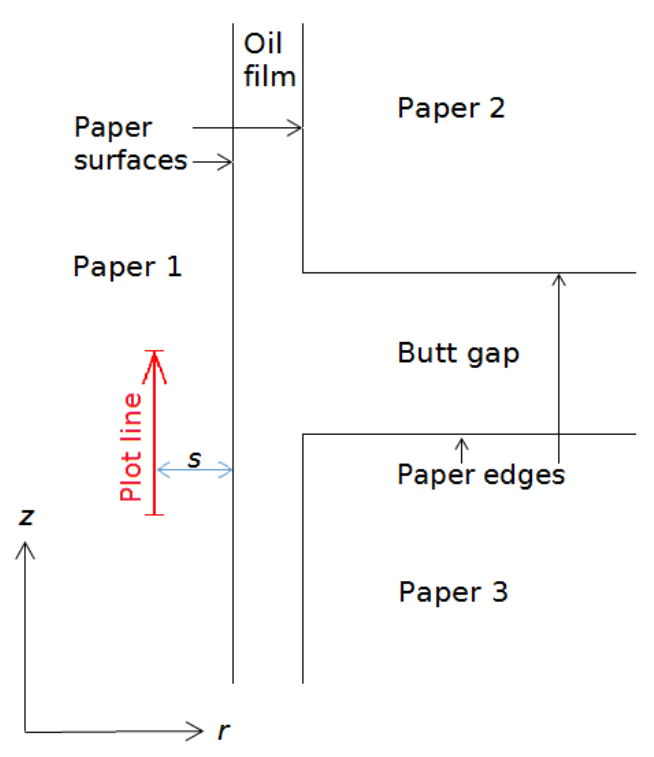

Fig. 4 - Definition of paper edges and the plot line. The plot line was placed in Paper 1, at various distances $s$ from its right surface. The distances in the $r$ direction are exaggerated in this figure.

each other in a similar manner as the field enhancements induced from butt gaps. This is visible in fig. 2 .

\subsection{Effect of film thickness}

Except for in areas of edge-induced field enhancements, a large oil film thickness led to a high field in the paper. The radial field was $7 \%$ higher if the oil film thickness was $10 \mu \mathrm{m}$ than if it was $1 \mu \mathrm{m}$. If no oil film was present, the radial field was $0.8 \%$ lower than if the oil film thickness was $1 \mu \mathrm{m}$. This can be seen in Figure 8 . The longitudinal field was zero in all cases, except for in areas with edgeinduced field enhancements.

In the areas affected by edge-induced field enhancements, the effect was opposite: A thicker film gave lower edgeinduced field enhancements. When calculations were done without any oil film between the paper layers, the paper corners produced field enhancement not only in the adjacent paper, but also in the paper corner itself. For example: The upper left corner of Paper 3 produced field enhancement in Paper 1. When there was no oil film between Paper 1 and Paper 3, the field enhancement was observed not only in Paper 1, but also in the corner of Paper 3.

\section{Discussion}

Even though $A$ in equation (7) was set to be 100 times higher for oil than for paper, $k=E_{\text {paper }} / E_{\text {oil }}$ was found to be not more than approximately 30 . This was due to the conductivity's dependence on the electric field strength.

The field enhancements from butt gaps in our model agreed well with the theory of Maxwell capacitors. With $k=30$, equation 5 predicted $24 \%$ enhancement when the butt gap and the areas to its left and right were considered

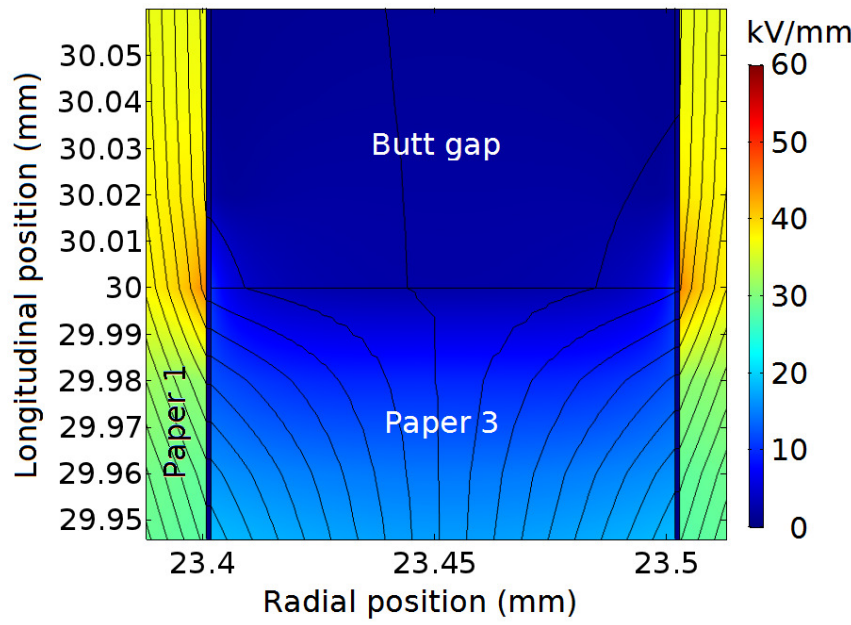

Fig. 5 - Magnitude of electric field (color surface plot) and equipotential lines (black) for each $0.1 \mathrm{kV}$. Film thickness: $1 \mu \mathrm{m}$. This figure is not stretched, so the scales of the $r$ and $z$ axes are equal.

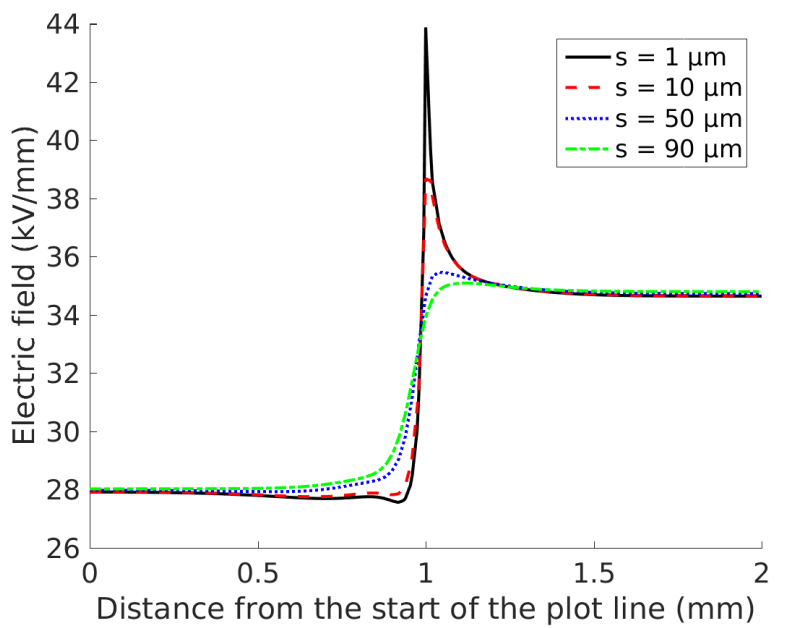

Fig. $6-E_{r}$ along the plot line (see Figure 4) at different distances $s$ from the paper surface. Film thickness: $1 \mu \mathrm{m}$.

as a Maxwell capacitor. Our results showed 24-25\% enhancement. The field enhancement from the butt gaps can also be understood simply by observing that the paper takes almost the entire voltage drop across the capacitor: Since there is less paper to take this voltage drop to the left and right of a butt gap, the field is higher such regions than in the rest of the paper.

As the number of paper layers increases, the effect of field enhancements becomes more important. Virtually the whole insulation of real cables are affected by field enhancements, and areas of coinciding field enhancements are normal. Some areas will probably suffer from coinciding enhancements from more butt gaps than other areas will, even though a sound production process will spread the butt gaps as much as possible. Changes of paper strip winding directions result in overlapping butt gaps and adds to this effect. The same discussion holds for edge-induced field enhancements. Even though 


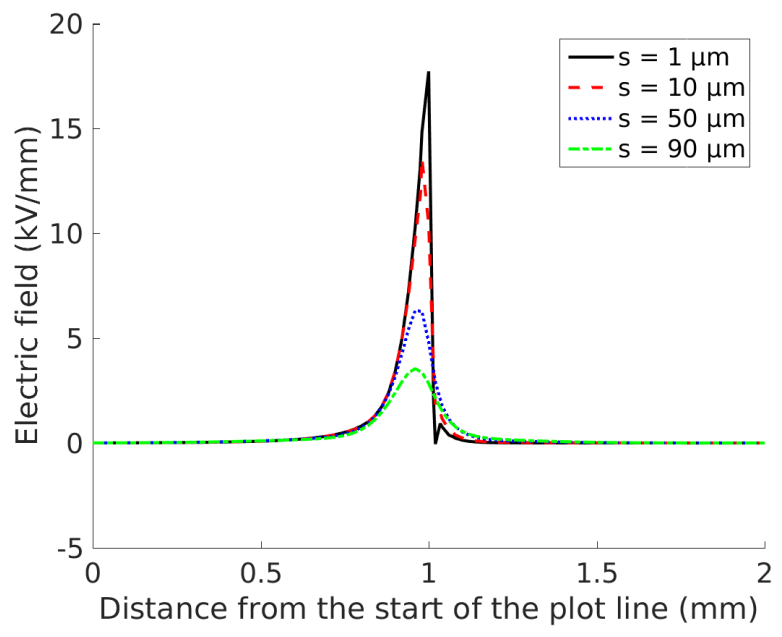

Fig. $7-E_{z}$ along the plot line (see Figure 4) at different distances $s$ from the paper surface. Film thickness: $1 \mu \mathrm{m}$.

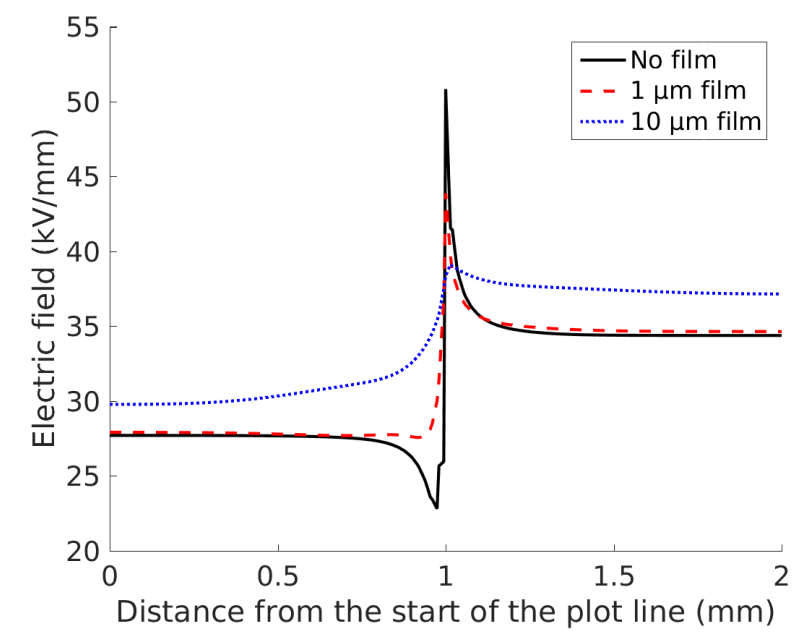

Fig. $8-E_{r}$ along the plot line (see Figure 4) at a distances $s=$ $1 \mu \mathrm{m}$ from the paper surface. No film, $1 \mu \mathrm{m}$ film, and $10 \mu \mathrm{m}$ film.

those enhancements diminish as the distance to the edge increases, they can add together, e.g. as shown in the middle of Paper 2 in Figure 2.

The field that we have calculated inside the paper is "macroscopic" in the sense that the effects of the internal heterogeneity of the paper is left out of the analysis. The heterogeneity, together with the surface roughness of the paper, may add further to the field enhancements in a real cable.

It can be argued that the paper-paper interfaces in reality are far more complex than an oil film with smooth surfaces. However, space charge measurements in stacked layers of mass-impregnated paper have yielded results consistent with the presence of $1-10 \mu \mathrm{m}$ oil films between the papers [5]. Nevertheless, emphasis should be on trends rather than absolute values in our calculationsespecially close to the surfaces.

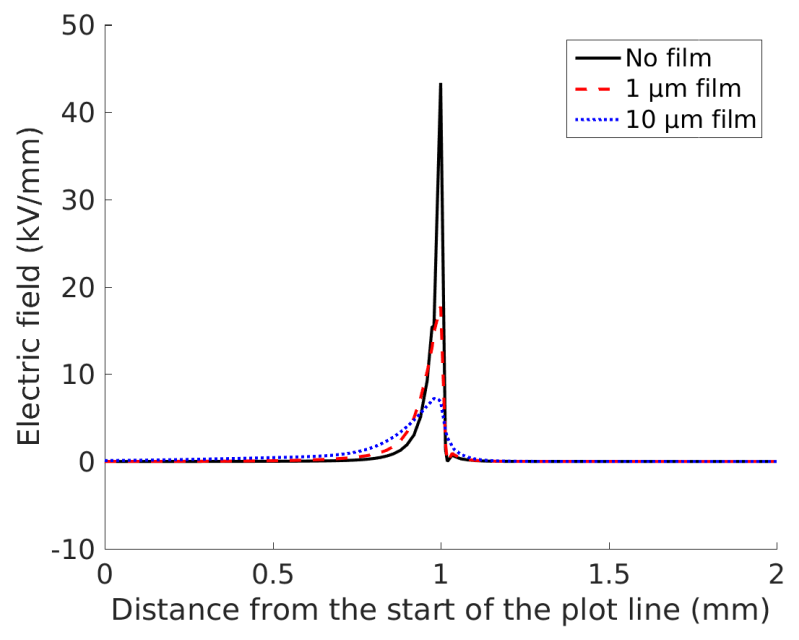

Fig. $9-E_{z}$ along the plot line (see Figure 4) at a distances $s=$ $1 \mu \mathrm{m}$ from the paper surface. No film, $1 \mu \mathrm{m}$ film, and $10 \mu \mathrm{m}$ film.

It is important to note that these considerations assume no cavities or other voids in the insulation.

\section{Conclusion}

In our model, the local electric field in the impregnated paper was equal to or higher than the macroscopic field. Butt gaps gave rise to field enhancements that reached far away from the butt gaps in the radial direction, but not in the longitudinal direction. Butt gaps with the same or almost the same longitudinal position intensified these enhancements.

The edges of papers and butt gaps gave rise to field enhancements that diminished as the distance from the edge increased. The only places in the model where a longitudinal component of the electric field was in such edge-induced enhancements. The radial component of the field was still the most dominant. Edges with the same or almost the same longitudinal position intensified these enhancements.

The field in the oil was negligible.

A thick oil film raised the field in the paper, except for close to paper edges where the edge-induced field enhancements were important.

\section{Acknowledgments}

This work is part of the project "Mass Impregnated Non-Draining HVDC Submarine Cables" led by Nexans Norway AS. The project is financially supported by The Research Council of Norway (Norges forskningsråd), Nexans Norway AS, Statnett SF, Affärsverket svenska kraftnät, and Fingrid Oyj, and has project number 256405/E20 within the ENERGIX program of The Research Council of Norway. 


\section{References}

[1] T. Worzyk. Submarine Power Cables: Design, Installation, Repair, Environmental Aspects. Springer Berlin Heidelberg, 2009.

[2] Nexans (press release). NSN Link will interconnect Nordic and British energy markets with the world's longest subsea power link incorporating Nexans' HVDC cable technology, July 2015.

[3] E. Occhini and G. Maschio. Electrical Characteristics of Oil-Impregnated Paper as Insulation for HV DC Cables. IEEE Transactions on Power Apparatus and Systems, PAS-86(3):312-326, March 1967.

[4] Bjørn R. Nyberg. Electrical stress in HVDC cables under stationary and transient conditions. $\mathrm{PhD}$ thesis, The University of Trondheim - The Norwegian Institute of Technology, Trondheim, 1973.

[5] M.J.P. Jeroense. Charges and discharges in HVDC cables: in particular in mass-impregnated HVDC cables. PhD thesis, Delft University Press, Delft, 1997.

[6] F. H. Kreuger. Industrial high DC voltage: 1. fields, 2. breakdowns, 3. tests. Delft Univ. Press, Delft, 1995.

[7] G. Evenset. Cavitation as a precursor to breakdown of mass-impregnated HVDC cables. $\mathrm{PhD}$ thesis, Norges tekniske høgskole, Institutt for elkraftteknikk, Trondheim, 1999.

[8] M. Runde, R. Hegerberg, N. Magnusson, E. Ildstad, and T. Ytrehus. Cavity formation in massimpregnated HVDC subsea cables-mechanisms and critical parameters. IEEE Electrical Insulation Magazine, 30(2):22-33, March 2014. 